\title{
CD205-TLR9-IL-12 axis contributes to CpG-induced oversensitive liver injury in HBsAg transgenic mice by promoting the interaction of NKT cells with Kupffer cells
}

\author{
Xin $\mathrm{Hou}^{1}$, Xiaolei $\mathrm{Hao}^{2}$, Meijuan Zheng ${ }^{3}$, Congfei Xu${ }^{4}$, Jun Wang ${ }^{2,4}$, Rongbin Zhou ${ }^{2,4}$ \\ and Zhigang Tian ${ }^{2,4}$
}

Gut-derived bacterial products contribute to liver inflammation and injury during chronic hepatitis B virus infection; however, the underlying mechanisms remain obscure. In this study, hepatitis B surface antigen transgenic (HBs-Tg) mice and their wild-type (WT) control C57BL/6 mice were injected with CpG-oligodeoxynucleotides (ODNs) to mimic the translocation of gut microbial products into the systemic circulation. We found that, compared with the WT mice, the HBs-Tg mice were oversensitive to CpG-ODN-induced liver injury, which was dependent on natural killer T (NKT) cells. CpG-ODN injection enhanced the expression of Fas ligand (FasL) on NKT cells. In addition, hepatocytes from the HBs-Tg mice expressed higher levels of Fas than did those from the WT mice, which was further augmented by CpG-ODN. Interaction of Fas and FasL was involved in the cytotoxicity of NKT cells against hepatocytes in the HBs-Tg mice. Moreover, Kupffer cells in the HBs-Tg mice expressed higher levels of CD205 and produced greater amounts of interleukin (IL)-12 than did those in the WT mice. Finally, the depletion of Kupffer cells, neutralization of IL-12 or specific silencing of CD205 on Kupffer cells significantly inhibited CpG-ODNinduced liver injury and NKT activation in the HBs-Tg mice. Our data suggest that CD205-expressing Kupffer cells respond to CpG-ODNs and subsequently release IL-12 to promote NKT cell activation. Activated NKT cells induce liver damage through the Fas signaling pathway in HBs-Tg mice. Cellular \& Molecular Immunology (2017) 14, 675-684; doi:10.1038/cmi.2015.111; published online 4 April 2016

Keywords: CpG; hepatitis B virus; Kupffer cell; liver injury; NKT cell

\section{INTRODUCTION}

More than 350 million people worldwide are persistently infected with hepatitis $\mathrm{B}$ virus (HBV) and are at risk of developing chronic active hepatitis, cirrhosis or cancer. One contributor to chronic inflammation and fibrosis in HBV infection is microbial translocation into the peripheral circulation. Gut-derived bacterial products, such as bacterial DNA and lipopolysaccharide (LPS), translocate into the portal system, where they are sensed and cleared by Kupffer cells. However, liver disease is often associated with Kupffer cell dysfunction, bacterial overgrowth and intestinal edema, resulting in increased amounts of microbial products in the systemic circulation. These elevated levels of microbial products may induce liver injury by activating Kupffer cells and other inflammatory signaling pathways. ${ }^{1,2}$ For example, in patients with an HBV infection, LPS-induced local and systemic inflammation are associated with severe liver fibrosis and are predictors of progression to end-stage liver disease. ${ }^{3}$ The presence of bacterial DNA in the ascitic fluid and serum of a patient with cirrhosis are indicators of a poor prognosis, which may be related to the development of acute-on-chronic liver failure in the short term. ${ }^{4}$ However, how bacterial products induce persistent inflammation and liver failure during $\mathrm{HBV}$ infection is not fully understood.

Unmethylated CpG motifs are found frequently in bacterial DNA, whereas they are underrepresented and methylated in vertebrate DNA; ${ }^{5}$ this marked difference between bacterial and vertebrate DNA enables vertebrate immune cells to detect and

\footnotetext{
${ }^{1}$ Anhui Provincial Laboratory of Microbiology and Parasitology, Department of Microbiology and Parasitology, Anhui Medical University, Hefei 230032, China; ${ }^{2}$ Institute of Immunology and CAS Key Laboratory of Innate Immunity and Chronic Disease, School of Life Sciences and Medical Center, University of Science and Technology of China, Hefei 230027, China; ${ }^{3}$ Department of Clinical Laboratory, First Affiliated Hospital of Anhui Medical University, Hefei 230022, China and ${ }^{4}$ Innovation Center for Cell Biology, Hefei National Laboratory for Physical Sciences at Microscale, Hefei 230027, China Correspondence: Professor Z Tian Ph.D., M.D., School of Life Sciences and Medical Center, University of Science and Technology of China, Hefei 230027, China. E-mail: tzg@ustc.edu.cn

Received: 20 September 2015; Revised: 7 October 2015; Accepted: 8 October 2015
} 
respond to bacterial infection. ${ }^{6}$ Synthetic oligodeoxynucleotides (ODNs) containing the unmethylated $\mathrm{CpG}$ motifs mimic the ability of either Gram-negative or Gram-positive bacterial DNA to activate innate immunity. ${ }^{7}$ It is well established that bacterial DNA and synthetic ODNs containing unmethylated CpG motifs are recognized by intracellular Toll-like receptor 9 (TLR9). ${ }^{8}$ CD205 is a recently identified cell surface receptor for CpG-ODN and is expressed in a variety of cells such as dendritic cells (DCs), B cells, T cells and macrophages. CD205 can directly bind CpG-ODNs and enhance their uptake. In the absence of CD205, DCs and B cells have shown impaired activation in response to CpG-ODNs, as well as diminished production of interleukin (IL)-12 or IL- $6{ }^{9}$

Kupffer cells are tissue-resident macrophages located in the liver sinusoid and form the largest population of immune cells in the liver. ${ }^{10}$ Owing to their abundance and location, Kupffer cells are the first barrier against pathogens entering the liver via the portal vein. They recognize pathogen-derived products via specific pattern recognition receptors, including TLRs, C-type lectins, Nod-like receptors and RIG-like receptors. ${ }^{11}$ Upon activation, Kupffer cells release large amounts of inflammatory cytokines such as IL-6, IL-12, IL-18, IL-1 $\beta$ and tumor necrosis factor (TNF). ${ }^{11}$ These cytokines are important for controlling the growth and dissemination of bacteria. However, excessive production of cytokines and dysregulated Kupffer cells may cause severe liver injury, ${ }^{12}$ especially in chronic HBV carriers, who are sensitive to hepatocyte damage. ${ }^{13}$ In addition, release of cytokines and chemokines by Kupffer cells is partially responsible for the recruitment and activation of other leukocytes such as natural killer (NK) cells and natural killer T (NKT) cells. ${ }^{14,15}$

NKT cells also express TLR9 and are activated and recruited to the liver by CpG-ODN treatment. ${ }^{16}$ During microbial infection, NKT cells become activated either through direct recognition of CD1d-presented microbial lipid antigens or by an innate cytokine- and self-antigen-driven pathway. ${ }^{17,18}$ After activation, NKT cells rapidly release remarkable quantities of cytokines such as IL-4 and IFN- $\gamma$. These cytokines have been found to take part in NKT cell-mediated liver injury. ${ }^{19}$ NKT cells also mediate liver injury through Fas-FasL signaling. ${ }^{20}$

In this study, using hepatitis B surface antigen (HBsAg) transgenic (HBs-Tg) mice that express HBsAg in serum, liver and kidney tissues to mimic human healthy chronic HBsAg carriers ${ }^{21}$ we found that the HBs-Tg mice were oversensitive to CpG-ODN-induced liver injury, which was absolutely dependent on hepatic NKT cells. The Fas-FasL signaling pathway was involved in the NKT cell-mediated liver injury in the HBs-Tg mice. Strikingly, Kupffer cells expressed high levels of CD205 in the HBs-Tg mice and promoted hepatic NKT cell activation via the production of IL-12 after CpG-ODN injection.

\section{MATERIALS AND METHODS}

\section{Animals}

The 8- to 10-week-old male HBs-Tg mice C57BL/6J-TgN (AlblHBV) 44Bri (H-2b, containing partial HBV genome, including $\mathrm{S}$, pre-S and $\mathrm{X}$ genes) were purchased from the Vital River experimental animal company (Beijing, China). The HBs-Tg mice were originally obtained from the Jackson Laboratory (Bar Harbor, ME, USA). C57BL/6 mice were also purchased from the Vital River experimental animal company. All mice were maintained in a specific pathogen-free microenvironment and received care in compliance with the guidelines outlined in the Guide for the Care and Use of Laboratory Animals. The protocols for all animal experiments were approved by the Institutional Animal Care and Use Committee at Anhui Medical University.

\section{CpG-ODN or non-CpG-ODN challenge}

Mouse CpG-ODN (HC4033: 5'-TCCATGACGTTCCTGA TGCT-3') and non-CpG-ODN control (HC4034: 5'-GCTT GATGACTCAGCCGGAA-3') were purchased from HyCult Biotechnology (Uden, Netherlands) and were dissolved in pyrogen-free saline. Mice were injected intravenously with $6 \mu \mathrm{g} / \mathrm{g}$ body weight of $\mathrm{CpG}$ or non-CpG.

\section{Hematoxylin and eosin staining}

Liver tissues were fixed in 10\% neutral buffered formalin and then embedded in paraffin. Tissue sections were affixed to slides, deparaffinized and stained with hematoxylin and eosin to determine morphologic changes.

\section{Cell depletion}

For NK1.1 $1^{+}$cell (including NK and NKT cells) depletion, mice received anti-NK1.1 monoclonal antibody (Ab; American Type Culture Collection, PK136) $24 \mathrm{~h}$ before being challenged. For NK cell depletion, mice were injected with $50 \mu \mathrm{g}$ of anti-ASGM1 Ab (Wako Co., Tokyo, Japan). Rabbit IgG (Sino-American Biotechnology Co., Beijing, China) was used as a control. To deplete Kupffer cells, gadolinium chloride ( $\mathrm{GdCl}_{3}$; Sigma Chemical Co., St. Louis, MO, USA) was intravenously injected at $10 \mathrm{mg} / \mathrm{kg}$ body weight $24 \mathrm{~h}$ before the following treatment.

\section{Isolation of liver mononuclear cells and NKT cells}

Liver mononuclear cells were isolated essentially as described previously. ${ }^{22}$ NKT cells were enriched by positive magnetic cell sorting using phycoerythrin (PE)-conjugated, PBS57-loaded CD1d tetramer and anti-PE-MicroBeads according to the manufacturer's protocol (Miltenyi Biotec, Auburn, CA, USA). The separated cells were $\geqslant 90 \%$ pure.

\section{Isolation of hepatocytes}

Each mouse was anesthetized and each portal vein was cannulated. Each liver was subsequently perfused with ethylene glycol tetraacetic acid solution $(5.4 \mathrm{mM} \mathrm{KCl}, 0.44 \mathrm{mM}$ $\mathrm{KH}_{2} \mathrm{PO}_{4}, 140 \mathrm{mM} \mathrm{NaCl}, 0.34 \mathrm{mM} \mathrm{Na}_{2} \mathrm{HPO}_{4}, 0.5 \mathrm{mM}$ ethylene glycol tetraacetic acid and $25 \mathrm{mM}$ tricine $(\mathrm{pH}$ 7.2)) and Dulbecco's modified Eagle medium (Gibco BRL, Gaithersburg, MD, USA) containing $0.075 \%$ collagenase I (Sigma-Aldrich, Shanghai, China). Then, the viable hepatocytes were separated by $40 \%$ Percoll (Gibco BRL) solution and centrifugation at $420 \mathrm{~g}$ for $10 \mathrm{~min}$. 


\section{Isolation of Kupffer cells}

Kupffer cells were isolated using a two-step collagenase perfusion method as described previously. ${ }^{22}$ The collected cells were allowed to attach to a plastic plate for $30 \mathrm{~min}$ for the removal of nonadherent cells. Then, cold phosphate-buffered saline (PBS) was added, and the cells were put on ice for $40 \mathrm{~min}$. After the dish was tapped gently, the supernatant was collected and centrifuged. The cells were resuspended in RPMI 1640 and immediately used.

\section{Flow cytometric analysis}

The fluorochrome-coupled monoclonal Abs used for flow cytometry in this study included CD69, FasL, Fas, CD3, NK1.1, CD19, F4/80, CD11c, CD11b, IgG2a, Rat IgG2b, ArH IgG2, ArH IgG1 (BD PharMingen, San Diego, CA, USA), granzyme B, perforin, CD205 and TLR9 (eBioscience, San Diego, CA, USA). The PE-conjugated, PBS57-loaded CD1d tetramer was a gift from the National Institutes of Health Tetramer Core Facility (Atlanta, GA, USA). After being incubated with Fc-blocker (2.4 G2; BD PharMingen), cells were stained with the indicated monoclonal Abs for surface antigens. Intracellular staining was performed using the Cytofix/Cytoperm Plus kit (BD Biosciences, San Diego, CA, USA) and Abs to TLR9, granzyme B and perforin. The stained cells were analyzed using a flow cytometer (FACScalibur; Becton Dickinson, Franklin Lakes, NJ, USA).

Analysis of cytokine and alanine aminotransferase levels Serum samples from mice were obtained at the indicated time points after CpG injection. Cytokine levels in the serum or the culture supernatants were measured using cytokine-specific enzyme-linked immunosorbent assay kits according to the manufacturer's instructions ( $\&$ D system, Minneapolis, $\mathrm{MN}$, USA). The serum alanine aminotransferase (ALT) levels were measured using a commercially available kit (Rong Sheng, Shanghai, China).

\section{Cytotoxicity assay}

NKT cell-mediated cytotoxicity against hepatocytes was measured by a 4 -h aspartate aminotransferase (AST) release assay. ${ }^{13}$ Hepatic NKT cells purified from the C57BL/6 mice as described above were added to the hepatocytes freshly isolated from the C57BL/6 or HBs-Tg mice at the indicated effector/ target cell ratios. After $4 \mathrm{~h}$, the supernatant was collected, and AST activity was measured. The specific cytotoxicity was calculated as follows:

$\left[\left(\mathrm{AST}_{\text {experimental }}-\mathrm{AST}_{\text {spontaneous }}\right) /\left(\mathrm{AST}_{\text {maximum }}-\mathrm{AST}_{\text {spontaneous }}\right)\right] \times 100 \%$

\section{Treatment of mice with nanoparticle-encapsulated siRNA}

To specifically silence the expression of CD205 on Kupffer cells in the HBs-Tg mice, siRNA targeting CD205 (siCD205, sense 5'-GCACUGGACACUGCUAAAUTT-3' and antisense 5'-AUU UAGCAGUGUCCAGUGCTT-3') was designed and synthetized by GenePharma (Shanghai, China). The negative control (siNeg) used was as follows: sense $5^{\prime}$-UUCUCCGAAC
GUGUCACGUTT-3' and antisense 5'-ACGUGACACGUU CGGAGAATT-3'). Cationic, lipid-assisted poly(ethylene glycol)-b-poly(d,l-lactide) (PEG-PLA) nanoparticles were used to encapsulate siCD205 or siNeg, and deliver them into Kupffer cells. ${ }^{15,23}$ Nanoparticle-encapsulated siCD205 or siNeg $(40 \mu \mathrm{g}$ per mouse) was administered to the mice $48 \mathrm{~h}$ before CpGODN treatment by intravenous injection.

\section{Statistical analysis}

Student's $t$-tests were used to compare values obtained from two groups. To compare values obtained from multiple groups, one-factor analysis of variance was used, followed by Tukey's post hoc test. All data are shown as the mean \pm s.e.m. $P$-values $<0.05$ were considered significant.

\section{RESULTS}

\section{HBs-Tg mice are more susceptible to CpG-induced liver} injury

CpG injection did not induce obvious liver injury in the C57BL/6 mice but induce severe liver damage in the HBs-Tg mice, as demonstrated by the elevation of serum ALT levels (Figure 1a), leukocyte infiltration and hepatocyte damage in the liver (Figure 1b). Compared with the C57BL/6 mice, the HBs-Tg mice showed significantly higher serum levels of IL-12 and TNF- $\alpha, 3$ and $1 \mathrm{~h}$ after CpG challenge, respectively (Figures $1 \mathrm{c}$ and $\mathrm{d}$ ). Both the HBs-Tg and C57BL/6 mice had comparable serum levels of IFN- $\gamma$ post CpG injection (Figure 1e).

\section{CpG-induced liver injury in HBs-Tg mice is NKT cell- dependent}

NKT cells were preferentially accumulated and activated in the liver after CpG-ODN injection in the C57BL/6 mice. ${ }^{16}$ To examine whether NKT cells contributed to the severe liver injury in the HBs-Tg mice after CpG injection, we depleted NK1.1 $1^{+}$cells (both NKT and NK cells) by treatment with antiNK1.1 and depleted NK cells with anti-AsGM1. As shown in Figure 2, depletion of both NKT cells and NK cells with antiNK1.1 significantly decreased serum ALT levels, leukocyte infiltration and hepatocyte damage, whereas depletion of NK cells alone with anti-ASGM1 did not affect serum ALT levels or cause liver histopathological changes. These results demonstrated that the liver injury induced by $\mathrm{CpG}$ in the HBs-Tg mice was dependent on NKT cells and not on NK cells.

Fas/FasL interaction is involved in NKT cell-mediated liver injury in HBs-Tg mice after CpG injection

To investigate the mechanisms underlying the role of NKT cells in CpG-induced liver injury in HBs-Tg mice, we first examined FasL expression on NKT cells. Because conventional $\mathrm{CD}^{+}$ $\mathrm{T}$ cells also express NK1.1 upon activation, we tracked NKT cells using PBS57-loaded CD1d tetramer in subsequent experiments. As shown in Figure 3a, the expression of FasL on NKT cells was upregulated in both the HBs-Tg and C57BL/6 mice after $\mathrm{CpG}$ treatment. However, $\mathrm{CpG}$ injection did not affect FasL expression on NK cells and T cells. Next, we examined the expression of Fas on hepatocytes. As illustrated in 


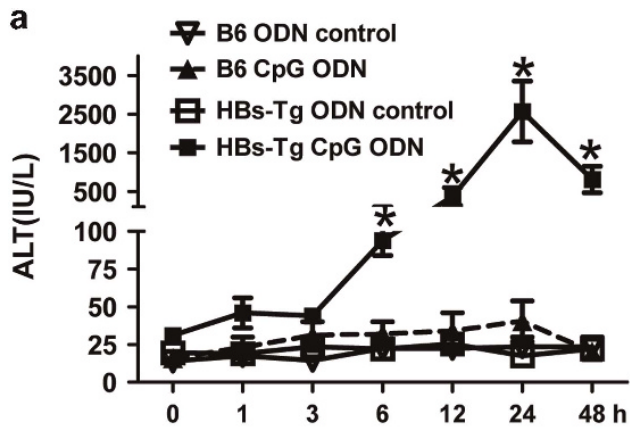

b ODN control
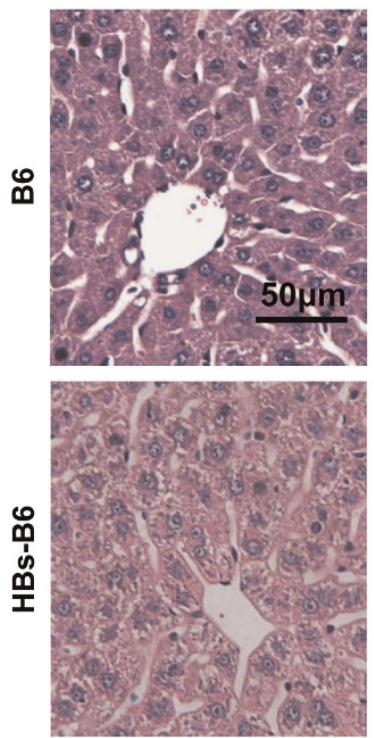

CpG ODN
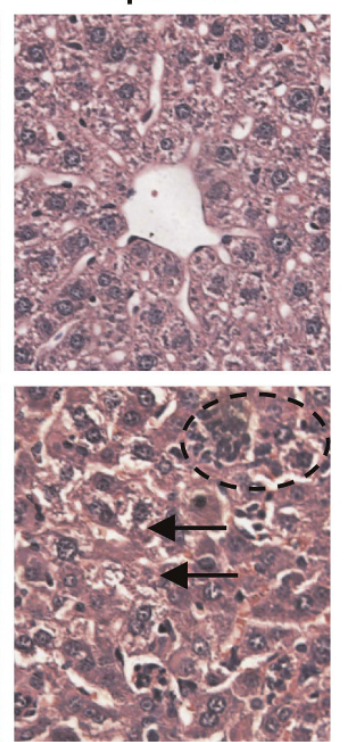

c

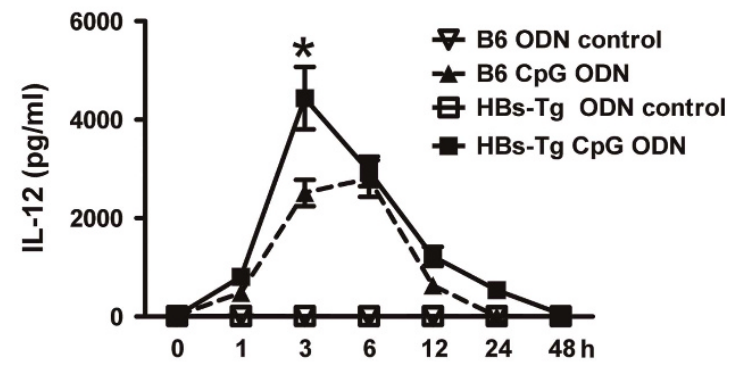

d
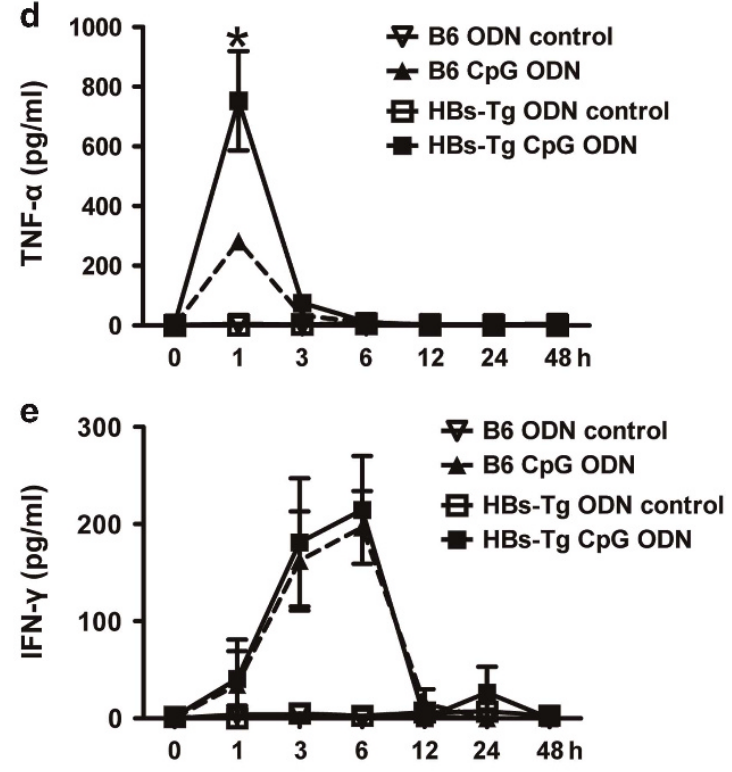

Figure $1 \mathrm{HBs}-\mathrm{Tg}$ mice are more susceptible to CpG-induced liver injury and cytokine production. HBs-Tg mice and C57BL/6 mice were injected intravenously with CpG (6 $\mu \mathrm{g} / \mathrm{g}$ body weight). (a) Serum ALT levels and (b) liver samples were collected $24 \mathrm{~h}$ after CpG challenge and then were prepared and stained with H\&E (original magnification $\times 200$ ). The arrows show hepatocyte damage and the circle shows leukocyte infiltration. (c-e) Serum cytokine levels were measured at the indicated time points after CpG or ODN control challenge. Values in $\mathbf{a}, \mathbf{c}, \mathbf{d}$ and $\mathbf{e}$ are shown as the mean \pm s.e.m. from five mice at each time point in each group and are from one representative experiment of two independent experiments. ${ }^{*} P<0.05$ compared with the other three groups (that is, B6 ODN control, B6 CpG ODN and HBs-Tg ODN control) determined by one-way ANOVA/Tukey's test. ALT, alanine aminotransferase; ANOVA, analysis of variance; HBs-Tg, hepatitis B surface antigen transgenic; H\&E, hematoxylin and eosin; ODN, oligodeoxynucleotide.

Figures $3 \mathrm{~b}$ and $\mathrm{c}$, higher levels of Fas expression were observed on hepatocytes of the HBs-Tg mice than on those of the C57BL/6 mice before and after $\mathrm{CpG}$ injection. Fas expression levels were increased in hepatocytes from both the HBs-Tg and C57BL/6 mice after CpG challenge.

To investigate whether the interaction between FasL and Fas contributed to the liver damage, we assessed direct cytotoxicity of the purified NKT cells against hepatocytes in a 4-h AST release assay in vitro (purity of the purified NKT cells is shown in Figure 4a). As shown in Figures $4 \mathrm{~b}$ and c, unstimulated NKT cells displayed little cytotoxicity against hepatocytes from the C57BL/6 and HBs-Tg mice (Figure 4b). However, CpGstimulated NKT cells showed greater cytotoxicity against hepatocytes from the C57BL/6 and HBs-Tg mice than did the unstimulated NKT cells (Figure 4c). The hepatocytes from the HBs-Tg mice were much more sensitive to CpG-stimulated NKT cell-induced cytotoxicity than those from the C57BL/6 mice (Figure 4c). Furthermore, incubation with FasL-blocking $\mathrm{Ab}$ diminished the cytotoxicity of NKT cells against hepatocytes from the HBs-Tg mice (Figure $4 \mathrm{~d}$ ). These data suggest that activated NKT cells induce hepatocyte damage via the FasL/Fas pathway. In addition, the production of perforin and granzyme B by NKT cells was also examined. CpG-induced perforin production by NKT cells in the HBs-Tg mice was higher than that in the $\mathrm{C} 57 \mathrm{BL} / 6$ mice, while granzyme $\mathrm{B}$ production induced by $\mathrm{CpG}$ was comparable in both the HBs-Tg and C57BL/6 mice (Supplementary Figure 1). These findings suggest that perforin may also contribute to CpG-induced liver injury in HBs-Tg mice.

NKT cell-mediated liver injury is Kupffer cell- and IL-12-dependent in HBs-Tg mice after CpG injection It has been reported that TLR-driven IL-12, rather than microbial antigens, is dominant in NKT cell activation during 


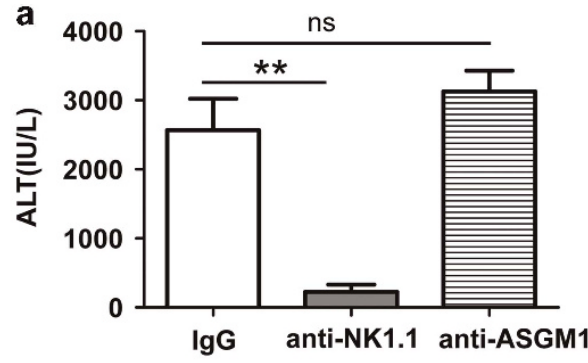

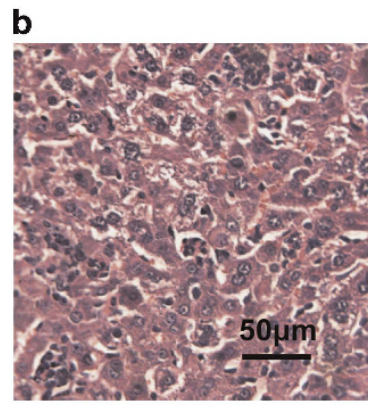

$\lg \mathbf{G}$

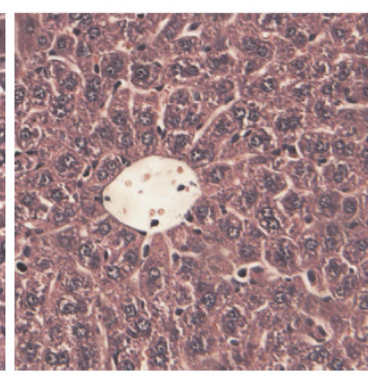

anti-NK1.1

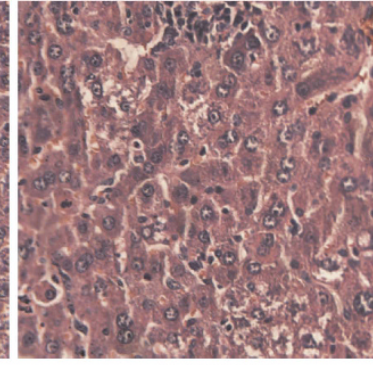

anti-ASGM1

Figure 2 Depletion of NKT cells attenuates liver injury induced by CpG in HBs-Tg mice. HBs-Tg mice were treated with control IgG, antiNK1.1 mAb (PK136) or anti-ASGM1 $24 \mathrm{~h}$ before CpG injection. Blood and liver tissue were collected $24 \mathrm{~h}$ after CpG injection. Liver injury was evaluated by (a) serum ALT and (b) H\&E staining of liver tissue (original magnification, $\times 200$ ). Data are representative of three independent experiments and are shown as the mean \pm s.e.m. ( $n=6$ per group). ${ }^{*} P<0.001$. ALT, alanine aminotransferase; HBs-Tg, hepatitis B surface antigen transgenic; H\&E, hematoxylin and eosin; IgG, immunoglobulin G; mAb, monoclonal antibody; NKT, natural killer T; NS, not significantly different.

a

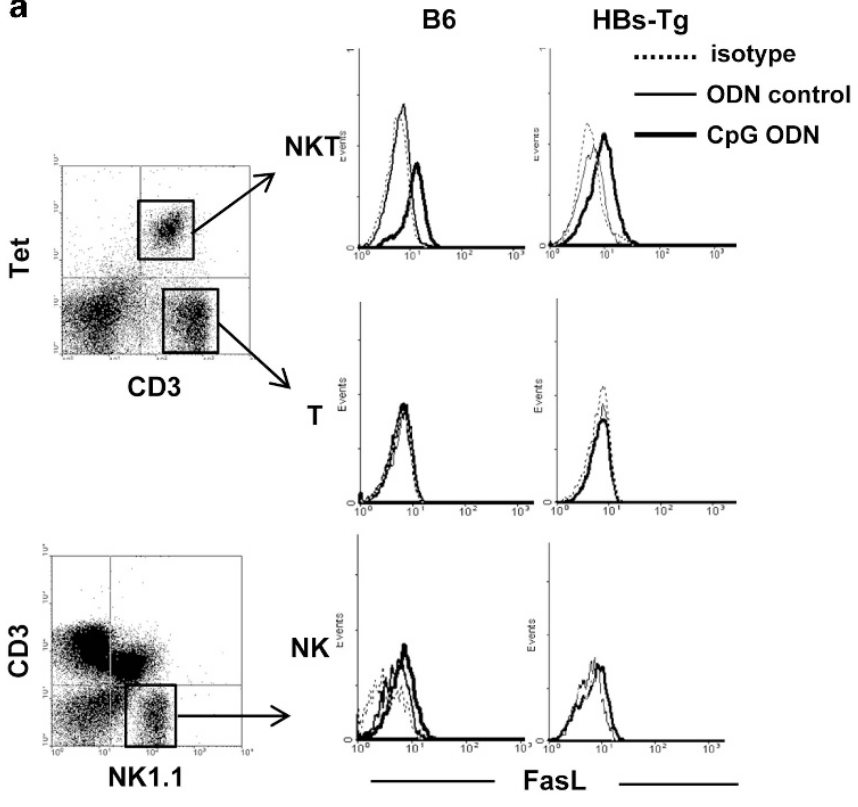

b
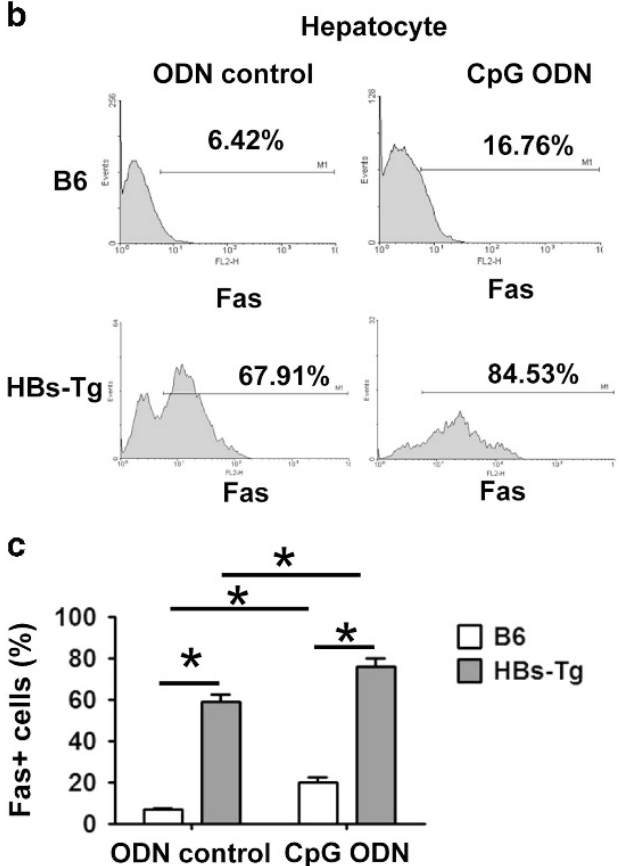

Figure $3 \mathrm{HBs}-\mathrm{Tg}$ mice are associated with upregulated expression of FasL on liver NKT cells and Fas on hepatocytes. C57BL/6 mice and HBs-Tg mice were treated with ODN control or CpG-ODN, and killed at $12 \mathrm{~h}$ after challenge. (a) Hepatic MNCs were isolated and examined by flow cytometry using anti-NK1.1, anti-CD3, PBS57-loaded CD1d tetramer and anti-FasL Abs. (b) Fas expression on hepatocytes was analyzed by flow cytometry. (c) Statistical analysis of the percentage of Fas-positive hepatocytes in b. Data are shown as the mean \pm s.e.m. ( $n=6$ mice per group) and are from one representative experiment of three independent experiments. ${ }^{*} P<0.05$. Ab, antibody; HBs-Tg, hepatitis B surface antigen transgenic; MNC, mononuclear cell; NKT, natural killer T; ODN, oligodeoxynucleotide. 
a

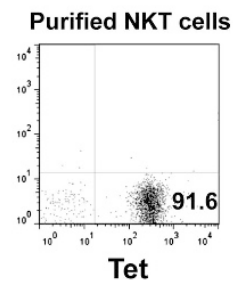

C

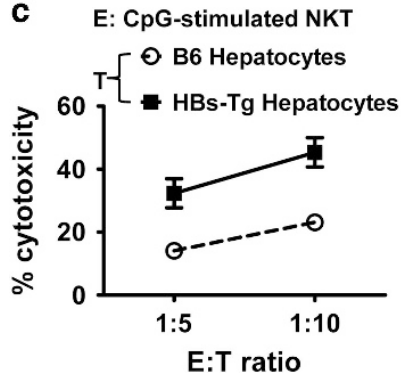

b

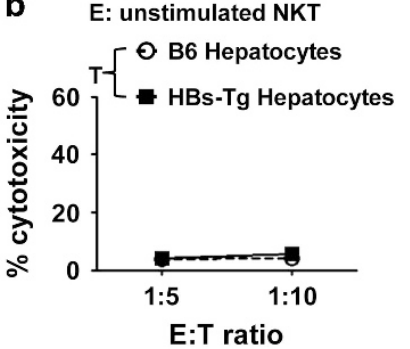

d

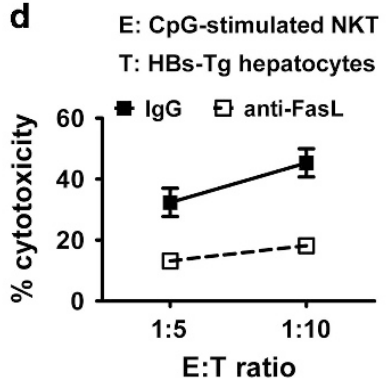

Figure 4 Fas/FasL interaction is involved in CpG-induced liver injury in HBs-Tg mice. (a) Representative graph showing the purity of sorted hepatic NKT cells (CD1d tetramer positive) from C57BL/6 mice; these NKT cells were used as effector cells. (b) Hepatic NKT cells were sorted from untreated C57BL/6 mice as effector cells. Hepatocytes isolated from untreated C57BL/6 mice or HBs-Tg mice were used as target cells. A cytotoxicity assay was performed. (c) Hepatic NKT cells were sorted from 6-h CpG-treated C57BL/6 mice. Hepatocytes were isolated from untreated C57BL/6 mice or HBs-Tg mice. (d) Hepatic NKT cells were sorted from 6-h CpGtreated $\mathrm{C} 57 \mathrm{BL} / 6$ mice. Hepatocytes isolated from untreated HBs-Tg mice were used as target cells. FasL-blocking antibody $\left(10 \mu \mathrm{gl}^{-1}\right)$ was added to the co-culture for the blockade. E/T, effector-target ratio; HBs-Tg, hepatitis B surface antigen transgenic; NKT, natural killer T.

microbial infection. ${ }^{18}$ We also observed large amounts of IL-12 production after $\mathrm{CpG}$ injection (Figure 1c). As Kupffer cells are the major source of IL-12, and they express TLRs, including TLR9, ${ }^{24,25}$ we then investigated the roles of Kupffer cells and IL-12 in CpG-induced liver injury in HBs-Tg mice. As illustrated in Figure 5a, depletion of Kupffer cells by pretreatment of $\mathrm{GdCl}_{3}$ markedly reduced CpG-induced liver injury in the HBs-Tg mice; neutralization of endogenous IL-12 by pretreatment with anti-IL-12 monoclonal Ab also ameliorated liver injury. Moreover, depletion of Kupffer cells or neutralization of IL-12 impaired the increases of FasL and CD69 expression on liver NKT cells induced by CpG-ODNs (Figure 5b). To confirm Kupffer cells as a major source of IL-12 after CpG challenge, we isolated Kupffer cells from ODN- or CpG-injected HBs-Tg mice and detected IL-12 production in the supernatants after $48 \mathrm{~h}$ of culture. We found that Kupffer cells from the CpG-injected HBs-Tg mice produced much greater amounts of IL-12 than did those from the ODN-treated mice (Figure 5c). Furthermore, Kupffer cell depletion by $\mathrm{GdCl}_{3}$ treatment significantly reduced serum levels of IL-12 in the HBs-Tg mice (Figure 5d).

Upregulation of CD205 expression on Kupffer cells from HBs-Tg mice

The above data suggest that Kupffer cells contribute to CpG-induced liver injury in $\mathrm{HBs}-\mathrm{Tg}$ mice by producing IL-12. To determine the reason that Kupffer cells from HBs-Tg mice produce more IL-12 than wild-type (WT) C57BL/6 mice after CpG stimulation, we examined the expression of extracellular and intracellular receptors of $\mathrm{CpG}$ in HBs-Tg and C57BL/6 mice. As shown in Figure 6, Kupffer cells from the HBs-Tg mice expressed significantly higher levels of CD205 than did those from the C57BL/6 mice, whereas expression levels of CD205 in hepatic DCs, B cells and peritoneal macrophages were comparable between the HBs-Tg and C57BL/6 mice. In addition, the TLR9 expression levels of these cells in the HBs-Tg and C57BL/6 mice were not significantly different.

\section{Specific silencing of CD205 in Kupffer cells alleviates $\mathrm{CpG}$-induced liver injury in $\mathrm{HBs}-\mathrm{Tg}$ mice}

To investigate whether the upregulated CD205 expression on Kupffer cells was responsible for the CpG-induced IL-12 production and liver injury in the HBs-Tg mice, we knocked down CD205 expression specifically in Kupffer cells, using nanoparticle-encapsulated CD205 siRNA, as reported in our previous studies. ${ }^{15,23}$ These nanoparticle-encapsulated CD205 siRNA significantly decreased CD205 expression on Kupffer cells, without affecting CD205 expression on hepatic DCs and hepatic B cells (Figures 7a and b). The silencing of CD205 in Kupffer cells significantly reduced serum levels of IL-12 (Figure 7c) and ALT (Figure 7d), and downregulated FasL and CD69 expression on hepatic NKT cells (Figure 7e) in the CpG-treated HBs-Tg mice. These data suggest that with the upregulated expression of CD205 in HBs-Tg mice, Kupffer cells capture more CpG and subsequently produce more IL-12.

\section{DISCUSSION}

Increased circulating levels of microbial products have been reported in patients with chronic viral hepatitis, and alcoholic and nonalcoholic fatty liver diseases. In addition, elevated plasma LPS or bacterial DNA levels have been noted as aggravating factors in these liver diseases. ${ }^{1-3}$ However, the underlying mechanisms remain poorly understood. Some studies using HBV transgenic mice have shown extraordinary sensitivity of the liver to immunologic and chemical triggers, such as Con A, polyI:C, $\mathrm{CCl}_{4}$ and a-galactosylceramide $(\alpha-G a l C e r)$, and demonstrated the contribution of NK/NKT cells to liver injury. ${ }^{26,27}$ To our knowledge, the role of Kupffer cells in the oversensitive injury of virus-infected livers remained unclear. In this study, we injected mice with CpG-ODNs to mimic the increased levels of microbial DNA in systemic circulation and thus explored the possible immunologic mechanisms of hepatocyte injury during HBV infection.

Kupffer cells are critical cellular components of the innate immune system in the liver. They specialize in scavenging and phagocytizing pathogen-derived products and apoptotic cells in blood. ${ }^{11}$ Here we demonstrated that Kupffer cells in HBs-Tg 
a

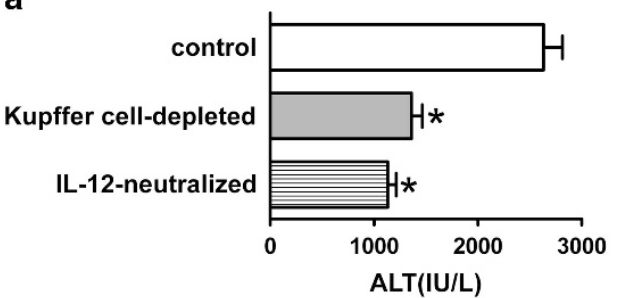

C Purified Kupffer cells

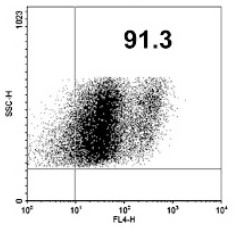

F4/80 b FasL

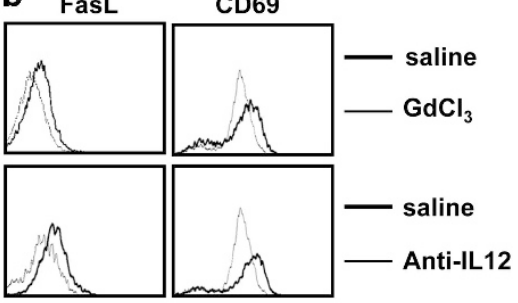

d

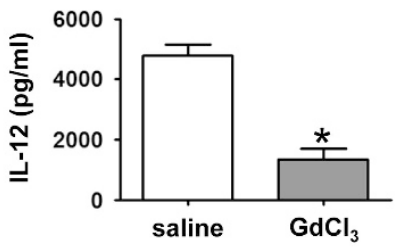

Figure 5 NKT cell-mediated liver injury is Kupffer cell- and IL-12-dependent in HBs-Tg mice after CpG injection. (a, b) Depletion of Kupffer cells was achieved by pretreatment with $\mathrm{GdCl}_{3} 24 \mathrm{~h}$ before $\mathrm{CpG}$ injection in HBs-Tg mice. Endogenous IL-12 was neutralized by pretreatment with anti-IL-12 mAb $12 \mathrm{~h}$ before CpG injection in HBs-Tg mice. Serum ALT levels were determined $24 \mathrm{~h}$ after CpG injection (a), and the expression levels of FasL and CD69 on hepatic NKT cells were determined by FACS analysis (b). (c) Kupffer cells were isolated from ODN control or CpG-ODN-treated HBs-Tg mice. After $48 \mathrm{~h}$ of culture, the supernatants were collected for IL-12 measurement by ELISA. (d) HBs-Tg mice were pretreated with $\mathrm{GdCl}_{3}$ or saline. Serum IL-12 levels were determined $3 \mathrm{~h}$ after $\mathrm{CpG}$ injection. All values are shown as the mean \pm s.e.m. from 6-8 mice per group and are representative of two experiments. ${ }^{*} P<0.05$ versus control group by Student's $t$-test. ALT, alanine aminotransferase; ELISA, enzyme-linked immunosorbent assay; FACS, fluorescence-activated cell sorting; HBs-Tg, hepatitis B surface antigen transgenic; IL, interleukin; mAb, antibody; NKT, natural killer T; ODN, oligodeoxynucleotide.
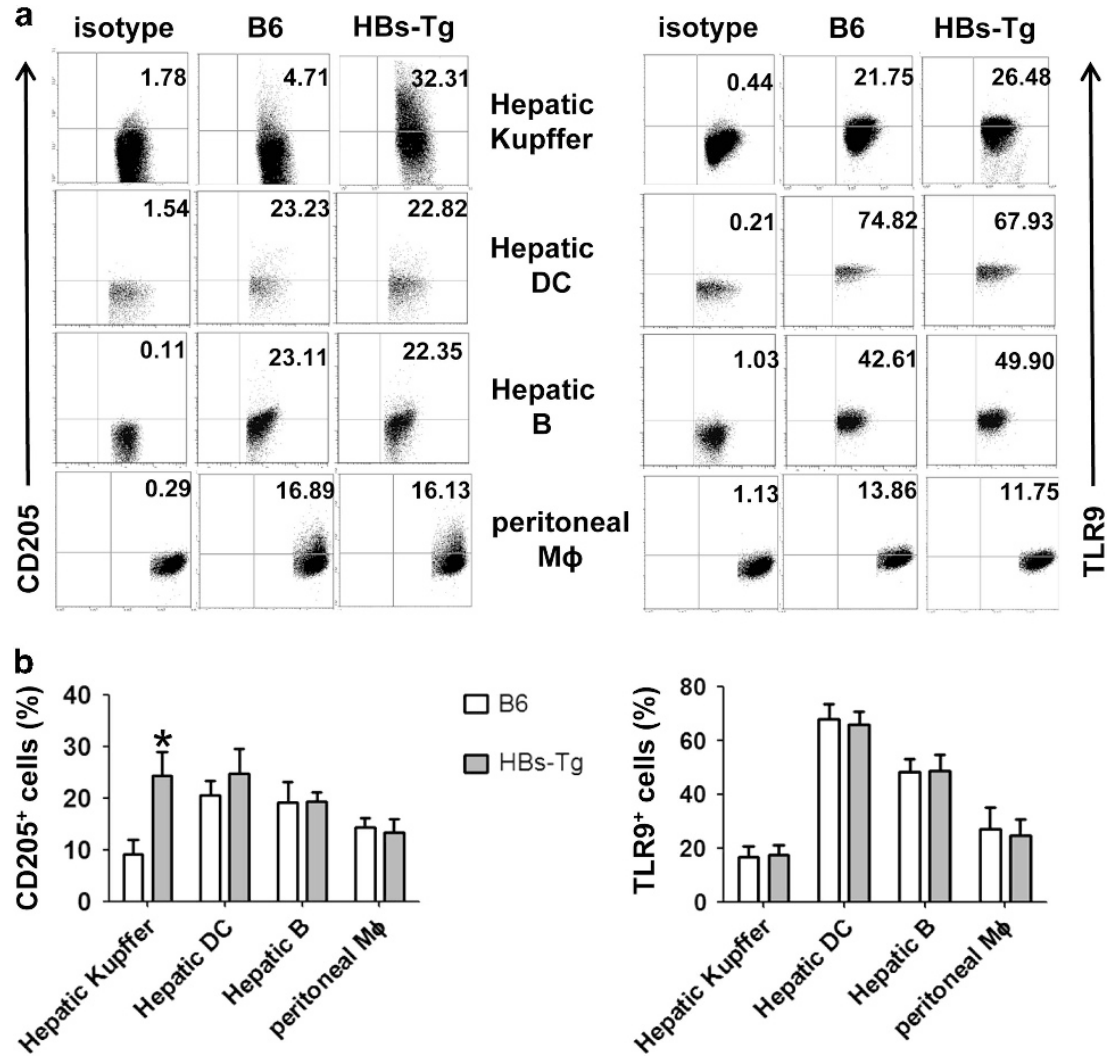

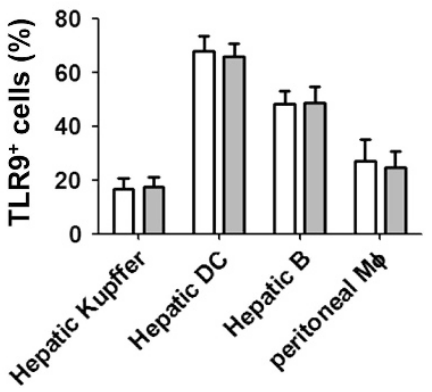

Figure 6 CD205 expression on hepatic immune cells and peritoneal macrophages in HBs-Tg and C57BL/6 mice. (a) Flow cytometry analysis of the expression of CD205 and TLR9 in Kupffer cells, hepatic DCs, hepatic B cells and peritoneal macrophages derived from HBs-Tg and C57BL/6 mice. (b) Graphical representation of the relative proportion of CD205 $5^{+}$cells and TLR9 ${ }^{+}$among various cells. Data are representative of three independent experiments and are shown as the mean \pm s.e.m. ( $n=6$ per group). ${ }^{*} P<0.05$ versus $\mathrm{C} 57 \mathrm{BL} / 6$ mice by unpaired, two-tailed Student's $t$-test. DC, dendritic cell; HBs-Tg, hepatitis B surface antigen transgenic; TLR9, Toll-like receptor 9. 

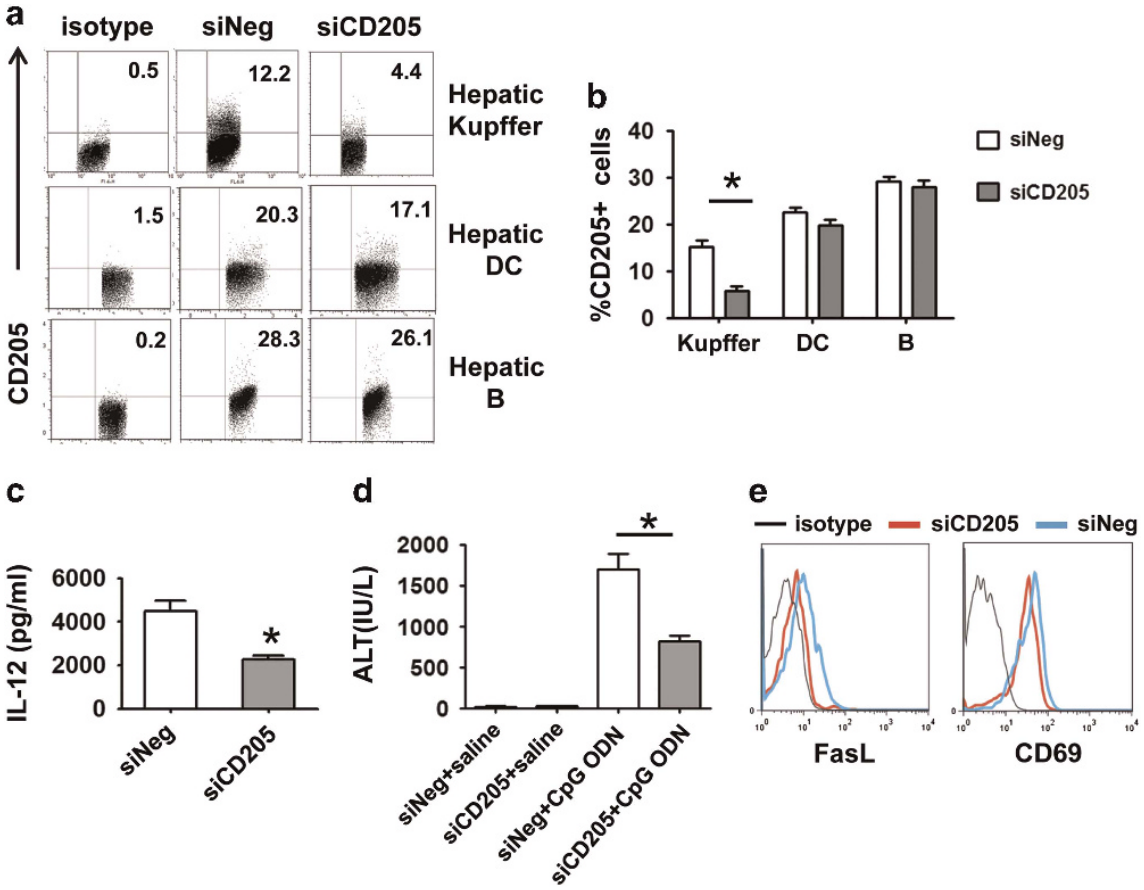

Figure 7 Silencing CD205 expression on Kupffer cells alleviates the liver injury induced by CpG in HBs-Tg mice. (a, b) HBs-Tg mice were treated with nanoparticle-encapsulated siCD205 or siNeg by intravenous injection; $48 \mathrm{~h}$ later, the expression of CD205 on various cells in the liver was examined by flow cytometry. (c-e) HBs-Tg mice were pretreated with nanoparticle-encapsulated siNeg or siCD205 $48 \mathrm{~h}$ before CpG challenge. Sera were collected $3 \mathrm{~h}$ after $\mathrm{CpG}$ challenge for IL-12 determination (c). Serum ALT levels were examined (d) and the expression of CD69 and FasL on hepatic NKT cells was analyzed by flow cytometry (e) from mice treated with CpG for 24 h. Data are representative of two independent experiments and are shown as the mean \pm s.e.m. $\left(n=4-6\right.$ per group). ${ }^{*} P<0.05$ versus siNeg-treated group by unpaired, two-tailed Student's $t$-test. ALT, alanine aminotransferase; HBs-Tg, hepatitis B surface antigen transgenic; IL, interleukin; NKT, natural killer T; siCD205, siRNA targeting CD205; siNeg, the negative control.

mice expressed higher levels of CD205 compared with those in WT mice (Figure 6). CD205 was newly identified as a critical receptor involved in the capture of CpG-ODNs in vivo. ${ }^{9}$ The upregulated expression of CD205 on Kupffer cells in HBs-Tg mice may be caused by HBsAg DNA stimulation or HBsAgassociated mild inflammation. It has been reported that $\mathrm{CD} 14^{+}$ intestinal macrophages, which are elevated in patients with Crohn disease, expressed higher levels of CD205 than CD14 ${ }^{-}$ typical intestinal resident macrophages. These CD $14^{+} \mathrm{CD} 205^{+}$ macrophages produced larger amounts of proinflammatory cytokines than $\mathrm{CD}_{14}{ }^{-} \mathrm{CD} 205^{-}$intestinal macrophages in response to commensal bacteria. ${ }^{28}$ We found that Kupffer cells in the HBs-Tg mice, which expressed higher levels of CD205, released more inflammatory cytokines than did those of the C57BL/6 mice. In addition, we found that Kupffer cells contributed to $\mathrm{CpG}$-induced liver injury in the HBs-Tg mice because the depletion of Kupffer cells or the silencing of CD205 on Kupffer cells markedly reduced IL-12 and ALT levels in the HBs-Tg mice (Figures $7 \mathrm{c}$ and $\mathrm{d}$ ).

In addition, the chemokines and cytokines produced by Kupffer cells have an important role in recruiting and activating other immune cells in the liver. It has been reported that Kupffer cell-secreted CXCL8 recruited NK and NKT cells to the liver during the early phase of HBV infection. ${ }^{14,29}$ Kupffer cells can also recruit DCs via C-type lectin interactions. ${ }^{30}$ This may result in complex interactions between Kupffer cells, infiltrating leukocytes and hepatocytes. Indeed, our results suggest a complex interaction between Kupffer cells and NKT cells in CpG-induced liver injury in HBs-Tg mice. Upon CpG stimulation, Kupffer cells produced a large amount of IL-12, which promoted the activation of NKT cells. When Kupffer cells were depleted, or IL-12 was neutralized, CpGODN-induced increases of CD69 and FasL expression on NKT cells were impaired (Figure 5b). In turn, activated NKT cells mediated hepatocyte damage via FasL/Fas interaction (Figure 4). Brigl et al. ${ }^{18}$ have also reported that TLR agonistinduced iNKT cell activation in the presence of DCs was driven by recognition of CD1d-presented self-antigens and IL-12, and diverse bacterial infection-induced activation of iNKT cells both in vitro and in vivo was overwhelmingly dependent on IL-12. Thus, antigen-presenting cell-derived IL-12 has an important role in promoting NKT cell activation.

The roles of NKT cells in HBV infection are complicated. Recent observations from patients with an HBV infection and animal models of HBV transgenic mice showed that NKT cells responded at very early time points after $\mathrm{HBV}$ exposure. ${ }^{31,32}$ NKT cell activation preceded the activation of NK cells and HBV-specific adaptive immune cells, and was associated with subsequent control over $\mathrm{HBV}$ infection. ${ }^{31,33} \mathrm{HBV}$ transgenic mice (expressing the complete HBV genome) treated with $\alpha$ GalCer exhibited increased IFN- $\gamma$ and IFN- $\alpha / \beta$ in the liver and decreased HBV replication that was dependent on NKT cells. ${ }^{34}$ 
However, $\alpha$-GalCer-activated NKT cells can also mediate liver injury in HBs-Tg mice. ${ }^{35}$ In a transgenic mouse model of primary HBV infection, nonclassical NKT cells mediated the development of acute hepatitis in an NKG2D-dependent manner. ${ }^{26}$ Our study suggested that NKT cells caused liver damage when exposed to CpG-DNA in HBs-Tg mice because the depletion of NKT cells markedly attenuated liver injury (Figure 2). Moreover, the interaction of Fas and FasL was involved in hepatic NKT cell-mediated hepatocyte damage (Figure 4). FasL is a type II membrane protein and belongs to the TNF family. It is predominantly expressed in activated T cells, NK cells and NKT cells. ${ }^{36}$ Our study showed that the increase of FasL expression was exclusively on hepatic NKT cells after $\mathrm{CpG}$ treatment (Figure 3a). We also compared the expression of Fas on hepatocytes between WT and HBs-Tg mice. Fas expression was obviously increased on hepatocytes in the HBs-Tg mice compared with the C57BL/6 mice (Figures $3 \mathrm{~b}$ and c), which caused the hepatocytes to be more sensitive to NKT cell-mediated cytotoxicity through Fas/FasL signaling after CpG triggering (Figure 4). Enhanced Fas expression in liver tissues has also been observed in patients with HBV- or hepatitis $\mathrm{C}$ virus-associated chronic liver diseases, ${ }^{37,38}$ and there was a progressive increase in its expression with the severity of the liver disease. Moreover, augmentation of the Fas system has been implicated in the pathogenesis of alcoholic liver disease, autoimmune hepatitis and biliary disorders. ${ }^{39,40}$ Thus, Fasmediated apoptosis has an important role in several liver diseases. In addition to upregulation of cell surface FasL expression, activated NKT cells have been shown to be capable of inducing hepatocyte death through the release of TNF- $\alpha$ and perforins/granzyme B. ${ }^{17}$ Our results showed increased expression of perforin in NKT cells derived from the HBs-Tg mice after $\mathrm{CpG}$ injection, suggesting that perforin may be involved in NKT cell-mediated liver injury in HBs-Tg mice.

Taken together, our findings suggest that the CD205-TLR9IL-12 axis has an important role in promoting CpG-mediated

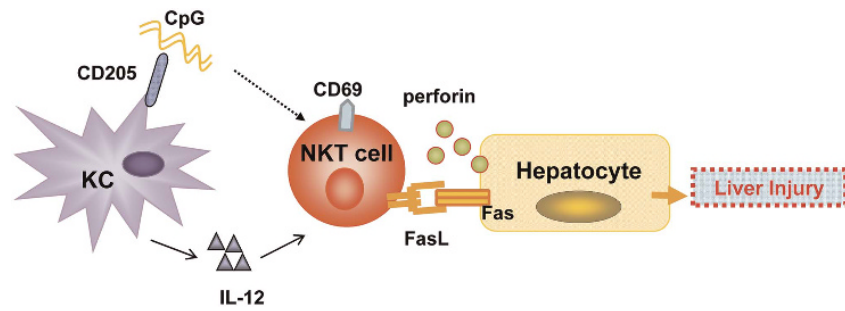

Figure 8 Model of the interaction of Kupffer cells and NKT cells triggered by $\mathrm{CpG}$ in mediating liver injury in $\mathrm{HBs}-\mathrm{Tg}$ mice. Kupffer cells from HBs-Tg mice express high levels of CD205 and produce great amounts of IL-12 after CpG stimulation. However, the mechanism underlying the upregulation of CD205 in Kupffer cells from HBs-Tg mice is not clear. Kupffer cell-derived IL-12 upregulates the expression of CD69 and FasL in NKT cells, resulting in enhanced cytotoxicity of NKT cells against hepatocytes via FasL/Fas interaction. In addition, activated NKT cells might induce hepatocyte death through the release of perforin. HBs-Tg, hepatitis B surface antigen transgenic; IL, interleukin; NKT, natural killer $\mathrm{T}$. liver injury in HBs-Tg mice by promoting the interaction of NKT cells with Kupffer cells (Figure 8). Kupffer cells from the HBs-Tg mice expressed higher levels of CD205 and produced greater amounts of cytokines (for example, IL-12) after CpG stimulation than did those from the C57BL/6 mice. Kupffer cell-derived IL-12 upregulated the expression of activation marker CD69 as well as FasL in NKT cells, resulting in enhanced NKT cell cytotoxicity against hepatocytes via FasL/Fas interaction. This study may help us better understand the roles of innate immune responses in the pathogenesis of microbial product-induced liver injury during $\mathrm{HBV}$ infection.

\section{CONFLICT OF INTEREST}

The authors declare no conflict of interest.

\section{ACKNOWLEDGEMENTS}

This work was supported by the Natural Science Foundation of China (81571540, 91429303, 81361120388, 31390433 and 81302525), the National Science \& Technology Major Projects (2013ZX10002002002) and the open project of the CAS Key Laboratory of Innate Immunity and Chronic Disease (KLIICD-201509).

1 Dolganiuc A, Norkina O, Kodys K, Catalano D, Bakis G, Marshall C et al. Viral and host factors induce macrophage activation and loss of toll-like receptor tolerance in chronic HCV infection. Gastroenterology 2007; 133: 1627-1636.

2 Harte AL, da Silva NF, Creely SJ, McGee KC, Billyard T, Youssef-Elabd EM et al. Elevated endotoxin levels in non-alcoholic fatty liver disease. J Inflamm (Lond) 2010; 7: 15.

3 Sandler NG, Koh C, Roque A, Eccleston JL, Siegel RB, Demino M et al. Host response to translocated microbial products predicts outcomes of patients with HBV or HCV infection. Gastroenterology 2011; 141: 1220-1230.

4 Zapater P, Frances R, Gonzalez-Navajas JM, de la Hoz MA, Moreu R, Pascual $S$ et al. Serum and ascitic fluid bacterial DNA: a new independent prognostic factor in noninfected patients with cirrhosis. Hepatology 2008; 48: 1924-1931.

5 Razin A FJ. DNA methylation and its possible biological roles. Prog Nucleic Acid Res Mol Biol 1981; 25: 33-52.

6 Krieg AM, Yi AK, Matson S, Waldschmidt TJ, Bishop GA, Teasdale R et al. CpG motifs in bacterial DNA trigger direct B-cell activation. Nature 1995; 374: 546-549.

7 Roman M, Martin-Orozco E, Goodman JS, Nguyen MD, Sato Y, Ronaghy A et al. Immunostimulatory DNA sequences function as T helper-1-promoting adjuvants. Nat Med 1997; 3: 849-854.

8 Ohto U, Shibata T, Tanji H, Ishida $\mathrm{H}$, Krayukhina E, Uchiyama S et al. Structural basis of $\mathrm{CpG}$ and inhibitory DNA recognition by Toll-like receptor 9. Nature 2015; 520: 702-705.

9 Lahoud MH, Ahmet F, Zhang JG, Meuter S, Policheni AN, Kitsoulis S et al. DEC-205 is a cell surface receptor for $\mathrm{CpG}$ oligonucleotides. Proc Natl Acad Sci USA 2012; 109: 16270-16275.

10 Jenne $\mathrm{CN}$, Kubes P. Immune surveillance by the liver. Nat Immunol 2013; 14: 996-1006.

11 Boltjes A, Movita D, Boonstra A, Woltman AM. The role of Kupffer cells in hepatitis B and hepatitis C virus infections. J Hepatol 2014; 61: 660-671.

12 Shen H, Sheng L, Chen Z, Jiang L, Su H, Yin L et al. Mouse hepatocyte overexpression of NF-kappaB-inducing kinase (NIK) triggers fatal macrophage-dependent liver injury and fibrosis. Hepatology 2014; 60: 2065-2076.

13 Chen Y, Wei H, Sun R, Dong Z, Zhang J, Tian Z. Increased susceptibility to liver injury in hepatitis $\mathrm{B}$ virus transgenic mice involves NKG2D-ligand interaction and natural killer cells. Hepatology 2007; 46: 706-715. 
14 Tu Z, Bozorgzadeh A, Pierce RH, Kurtis J, Crispe IN, Orloff MS. TLR-dependent cross talk between human Kupffer cells and NK cells. J Exp Med 2008; 205: 233-244.

15 Cui K, Yan G, Xu C, Chen Y, Wang J, Zhou R et al. Invariant NKT cells promote alcohol-induced steatohepatitis through interleukin-1beta in mice. J Hepatol 2015; 62: 1311-1318.

16 Jiang W, Sun R, Zhou R, Wei H, Tian Z. TLR-9 activation aggravates concanavalin A-induced hepatitis via promoting accumulation and activation of liver CD4+ NKT cells. J Immunol 2009; 182: 3768-3774.

17 Santodomingo-Garzon T, Swain MG. Role of NKT cells in autoimmune liver disease. Autoimmun Rev 2011; 10: 793-800.

18 Brigl M, Tatituri RV, Watts GF, Bhowruth V, Leadbetter EA, Barton N et al. Innate and cytokine-driven signals, rather than microbial antigens, dominate in natural killer $\mathrm{T}$ cell activation during microbial infection. J Exp Med 2011; 208: 1163-1177.

19 Kaneko Y, Harada M, Kawano T, Yamashita M, Shibata Y, Gejyo F et al. Augmentation of Valpha14 NKT cell-mediated cytotoxicity by interleukin 4 in an autocrine mechanism resulting in the development of concanavalin A-induced hepatitis. J Exp Med 2000; 191: 105-114.

20 Takeda K, Hayakawa Y, Van Kaer L, Matsuda H, Yagita H, Okumura K. Critical contribution of liver natural killer T cells to a murine model of hepatitis. Proc Natl Acad Sci USA 2000; 97: 5498-5503.

21 Chisari FV, Filippi P, McLachlan A, Milich DR, Riggs M, Lee S et al. Expression of hepatitis $B$ virus large envelope polypeptide inhibits hepatitis B surface antigen secretion in transgenic mice. J Virol 1986; 60: 880-887.

22 Hou X, Zhou R, Wei H, Sun R, Tian Z. NKG2D-retinoic acid early inducible-1 recognition between natural killer cells and Kupffer cells in a novel murine natural killer cell-dependent fulminant hepatitis. Hepatology 2009; 49: 940-949.

$23 \mathrm{Li} \mathrm{Y,} \mathrm{Cao} \mathrm{G,} \mathrm{Zheng} \mathrm{X,} \mathrm{Wang} \mathrm{J,} \mathrm{Wei} \mathrm{H,} \mathrm{Tian} \mathrm{Z} \mathrm{et} \mathrm{al.} \mathrm{CRACC-}$ CRACC interaction between Kupffer and NK cells contributes to poly I:C/D-GalN induced hepatitis. PLoS One 2013; 8: e76681.

24 Chen Y, Sun R. Toll-like receptors in acute liver injury and regeneration. Int Immunopharmacol 2011; 11: 1433-1441.

25 Seki E, Brenner DA. Toll-like receptors and adaptor molecules in liver disease: update. Hepatology 2008; 48: 322-335.

26 Vilarinho S, Ogasawara K, Nishimura S, Lanier LL, Baron JL. Blockade of NKG2D on NKT cells prevents hepatitis and the acute immune response to hepatitis B virus. Proc Natl Acad Sci USA 2007; 104: 18187-18192.

27 Chen Y, Sun R, Wu X, Cheng M, Wei H, Tian Z. CD4+CD25+ regulatory $T$ cells inhibit natural killer cellhepatocytotoxicity of hepatitis $B$ virus transgenic mice via membrane-bound TGF-beta and OX40. J Innate Immun 2016; 8: 30-42.

28 Kamada N, Hisamatsu T, Okamoto S, Chinen H, Kobayashi T, Sato $\mathrm{T}$ et al. Unique CD14 intestinal macrophages contribute to the pathogenesis of Crohn disease via IL-23/IFN-gamma axis. J Clin Invest 2008; 118: 2269-2280.

29 Hosel M, Quasdorff M, Wiegmann K, Webb D, Zedler U, Broxtermann M et al. Not interferon, but interleukin-6 controls early gene expression in hepatitis B virus infection. Hepatology 2009; 50: 1773-1782.

30 Uwatoku R, Suematsu M, Ezaki T, Saiki T, Tsuiji M, Irimura T et al. Kupffer cell-mediated recruitment of rat dendritic cells to the liver: roles of $\mathrm{N}$-acetylgalactosamine-specific sugar receptors. Gastroenterology 2001; 121: 1460-1472.

31 Zeissig S, Murata K, Sweet L, Publicover J, Hu Z, Kaser A et al. Hepatitis B virus-induced lipid alterations contribute to natural killer T cell-dependent protective immunity. Nat Med 2012; 18: 1060-1068.

32 Webster GJ, Reignat S, Maini MK, Whalley SA, Ogg GS, King A et al. Incubation phase of acute hepatitis $B$ in man: dynamic of cellular immune mechanisms. Hepatology 2000; 32: 1117-1124.

33 Fisicaro P, Valdatta C, Boni C, Massari M, Mori C, Zerbini A et al. Early kinetics of innate and adaptive immune responses during hepatitis $\mathrm{B}$ virus infection. Gut 2009; 58: 974-982.

34 Kakimi K, Guidotti LG, Koezuka Y, Chisari FV. Natural killer T cell activation inhibits hepatitis $\mathrm{B}$ virus replication in vivo. J Exp Med 2000; 192: 921-930.

35 Trobonjaca Z, Kroger A, Stober D, Leithauser F, Moller P, Hauser H et al. Activating immunity in the liver. II. IFN-beta attenuates NK celldependent liver injury triggered by liver NKT cell activation. J Immunol 2002; 168: 3763-3770

36 Lettau M, Paulsen M, Kabelitz D, Janssen O. Storage, expression and function of Fas ligand, the key death factor of immune cells. Curr Med Chem 2008; 15: 1684-1696.

37 Bortolami M, Kotsafti A, Cardin R, Farinati F. Fas/FasL system, IL-1beta expression and apoptosis in chronic HBV and HCV liver disease. J Viral Hepat 2008; 15: 515-522.

38 Oksuz M, Akkiz H, Isiksal YF, Saydaogluc G, Serin M, Kayaselcuk F et al. Expression of Fas antigen in liver tissue of patients with chronic hepatitis B and C. Eur J Gastroenterol Hepatol 2004; 16: 341-345.

39 Tagami A, Ohnishi H, Moriwaki H, Phillips M, Hughes RD. Fasmediated apoptosis in acute alcoholic hepatitis. Hepatogastroenterology 2003; 50: 443-448.

40 Seki S, Kitada T, Iwai S, Kadoya H, Yamada T, Kawada N et al. Immunohistochemical detection of Fas and apoptosis in type-1 autoimmune hepatitis. Hepatogastroenterology 2003; 50: $1274-1277$.

(c) (1) () $\odot$ This work is licensed under a Creative Commons Attribution-NonCommercial-NoDerivs 4.0 International License. The images or other third party material in this article are included in the article's Creative Commons license, unless indicated otherwise in the credit line; if the material is not included under the Creative Commons license, users will need to obtain permission from the license holder to reproduce the material. To view a copy of this license, visit http://creativecommons.org/licenses/by-nc-nd/4.0/

Supplementary Information for this article can be found on the Cellular \& Molecular Immunology website (http://www.nature.com/cmi) 